\title{
Tracheal stenosis due to huge adenomatous goiter
}

\author{
Takeshi Kusunoki ${ }^{* 1}$, Hirotomo Homma ${ }^{1}$, Yoshinobu Kidokoro ${ }^{1}$, Aya Yanai ${ }^{1}$, Ryo Wada ${ }^{2}$ and Katsuhisa Ikeda ${ }^{3}$ \\ ${ }^{1}$ Department of Otorhinolaryngology, Juntendo University of Medicine, Shizuoka Hospital, Japan \\ ${ }^{2}$ Department of Pathology, Juntendo University of Medicine, Shizuoka Hospital, Japan \\ ${ }^{3}$ Department of Otorhinolaryngology, Juntendo University of Medicine, Faculty of Medicine, Japan
}

\begin{abstract}
Background: Some thyroid anaplastic carcinoma and lymphoma have been reported to cause tracheal stenosis or choking. Begin thyroid tumors with dyspnea due to tracheal stenosis are exceedingly rare.

Case presentation: We experienced a huge adenomatous goiter (resected specimen weight: 520g). Total thyroidectomy resolved the dyspnea and there was no tracheomalacia. Postoperative CT confirmed a normal tracheal lumen free from stenosis.

Conclusions: We reviewed the CT findings of thyroid benign tumors with dyspnea and found that tumors occupied the space between the posterior wall of trachea and esophagus leading to tracheal stenosis as in our case. Such tracheal findings would actually lead to dyspnea.
\end{abstract}

\section{Introduction}

Some thyroid malignant tumors and thyroid malignant lymphoma have been reported to cause tracheal stenosis [1,2]. Benign thyroid tumors with dyspnea due to tracheal stenosis are very rare [3]. We experienced a case of a huge benign tumor (resected specimen weight: $520 \mathrm{~g}$ ) with dyspnea due to tracheal stenosis and performed total thyroidectomy. This case report presents the clinical findings, imaging examination, operation and postoperative progress. Moreover, we discuss the tracheal stenosis pattern with dyspnea by referring to various studies.

\section{Case presentation}

A 54-year-old Japanese woman consulted our hospital with dyspnea at neck flexion and when supine. Therefore, she went to bed in neck retroflection. In the initial findings, both lobes of the thyroid were diffusely enlarged to the lower jaw (Figure 1). In the CT scan, bilateral lobes of the thyroid tumors were diffusely swollen and had grown into the space between the posterior wall of the trachea and esophagus, leading to the tracheal stenosis by compressing the posterior wall of the trachea (Figure 2). On the blood examination, thyroid function, microsome test, and thyroid test were within normal limits. Thyroglobulin $(729.6 \mathrm{ng} / \mathrm{ml}$ ) was high (normal range: 0-33 ng/ml).

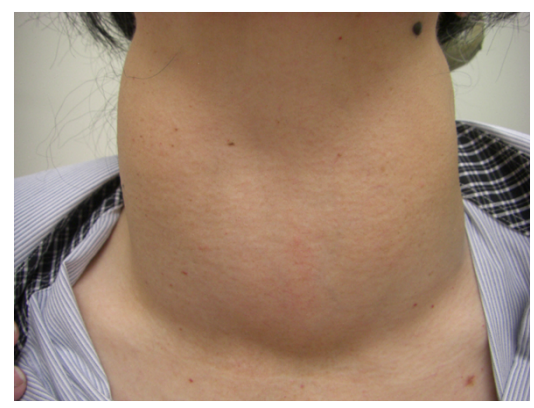

Figure 1. A thyroid tumor was diffuse swelling that reached to the lower jaw in the bilateral superior pair of thyroid lobes.
We performed total thyroidectomy with preservation of the bilateral recurrent nerve, main blood vessels and thyroid capsule. However, oozing from the entire thyroid surface continued during surgery. The operation time was 6 hours and the bleeding volume was $1450 \mathrm{~g}$. The size and weight of the enblock resected specimen was $13 \times$ $11 \times 6 \mathrm{~cm}$ and $520 \mathrm{~g}$ (Figure 3). The postoperative histopathology was adenomatous goiter without malignant cells and chronic thyroiditis (Figure 4). The progress after surgery was good, and there was no dyspnea, hemorrhage or vocal cord paralysis. In postoperative CT, the trachea appeared to be free from stenosis and normal (Figure 5). Postoperative thyroglobulin $(10.2 \mathrm{ng} / \mathrm{ml})$ was within the normal range.

\section{Discussion}

Huge thyroid malignant lymphoma with dyspnea has been reported

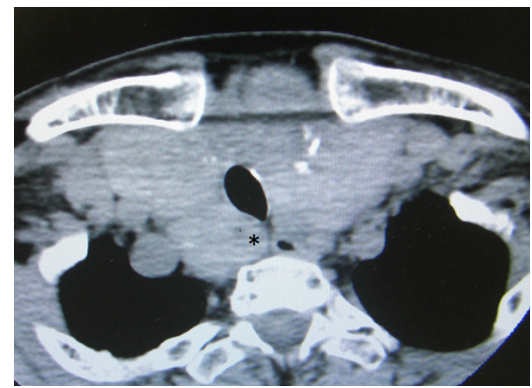

Figure 2. On preoperative CT, bilateral lobes of the thyroid tumors were diffusely enlarged and occupied the space (asterisk) between the posterior wall of the trachea and esophagus leading to tracheal stenosis.

Correspondence to: Dr. Takeshi Kusunoki, Department of Otorhinolaryngology, Juntendo University of Medicine, Shizuoka Hospital 1129 Nagaoka Izunokunishi, Shizuoka 410-2295, Japan; Fax: +81-55-948-5088; E-mail: ttkusunoki001@aol.com

Key words: tracheal stenosis, dyspnea, begin thyroid tumors, adenomatous goiter

Received: December 20, 2016; Accepted: January 13, 2017; Published: January 20, 2017 


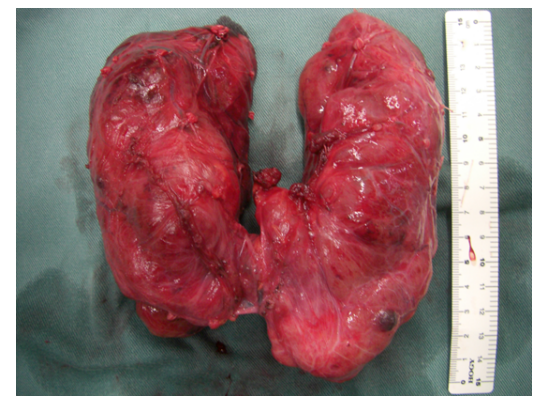

Figure 3. The weight of the enblock resected specimen was $520 \mathrm{~g}$.

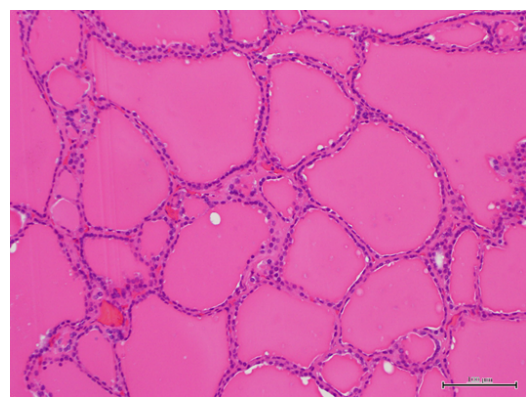

Figure 4. Postoperative histopathological examination showed an adenomatous goiter without malignant cells and chronic thyroiditis.

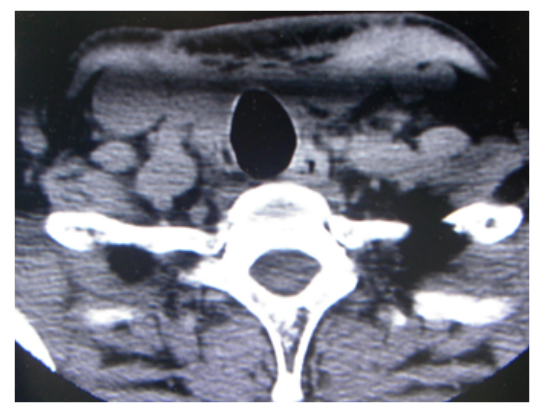

Figure 5. Left: The neck swelling disappeared after total thyroidectomy

Right: In the postoperative $\mathrm{CT}$, the trachea was normal and free from stenosis.

and Nomizu [4] presented a case with choking. Thyroid lymphoma occurs in chronic thyroiditis [5]. Sato [6] experienced a case of chronic thyroiditis without malignant lymphoma that caused narrowing of the air way, for which emergency tracheotomy was performed. Alfonso [7] demonstrated that the incidence of tracheoesophageal compression in thyroid benign diseases with chronic thyroiditis was $67 \%$. In our case, the histopathological diagnosis was an adenomatous goiter without chronic thyroiditis or thyroid dysfunction, and the microsome and thyroid test results were within normal limits in the preoperative blood examination.

Not all huge thyroid benign tumors with tracheal compression could cause dyspnea. We reviewed the CT findings of thyroid benign tumors with dyspnea In an found that they occupied the space between the posterior wall of the trachea and esophagus leading to tracheal stenosis as our case $[6,8]$. The above tracheal findings would actually lead to dyspnea.

Agarwal [9] described that the incidence of tracheomalacia with huge goiters was high. Some hospitals performed tracheostomy and inserted a T-tube into the trachea lumen after thyroidectomy for huge thyroid benign tumors $[8,10]$. In our case we observed no clinical findings of tracheomalacia after surgery. Postoperative CT confirmed a normal tracheal lumen without stenosis.

\section{Declarations}

\section{Author's contributions}

Takeshi Kusunoki MD: diagnosis and therapy, composition of this case report, Hirotomo Honma MD: therapy, Yoshinobu Kidokoro MD: therapy, Aya Yanai MD: therapy, Ryo Wada MD: Pathological diagnosis, Katsuhisa Ikeda MD: composition of this case report

\section{Consent for publication}

Consent for publication on the clinical details, images and histopathological examination was obtained for this case report. A copy of this document is available for review by the Editor of this journal.

\section{Acknowledgements}

The authors thank Mr. Brent Bell for checking English of the manuscript.

\section{References}

1. Lawson W, Som HL, Biller HF (1977) Papillary adenocarcinoma of the thyroid invading the upper air passages. Ann Otol Rhinol Laryngol 86: 751-755.[Crossref]

2. Taura M, Kamizono K, Ejma M, Hara T, Nakano R, et al. (2010)Primay thyroid lymphoma: A report of three cases. Otologia Fukuoka 56: 243-246.

3. Jauregui R, Lilker ES, Bayley A (1977) Upper airway obstruction in euthyroidgoiter. JAMA 238: 2163-2166.[Crossref]

4. Nomizu T, Matsuzaki M, Katagata N, Kanke Y, Sakuma T, etal. (2014) A case of malignant lymphoma of the neck which presented the huge struma. JJAES JSTS 31: 134-138.

5. Matsuzuka F, Miyauchi A, Katayama S, Narabayashi I, Ikeda H, et al. (1993) Clinical aspects of primary thyroid lymphoma: diagnosis and treatment based on our experience of 119 cases. Thyroid3: 93-99.[Crossref]

6. Sato K, Hanazawa H, Watanabe J, Takahashi S (2005) Differential diagnosis and management of airway obstruction in Riedel's thyroiditis: a case report. AurisNasus Larynx 32: 439-443.[Crossref]

7. Alfonso A, Christoudias G, Amaruddin Q, Herbsman H, Gardner B (1981) Tracheal or esophageal compression due to benign thyroid disease. Am J Surg 142: 350-354. [Crossref]

8. Ayabe H, Kawahara K, Tagawa Y, Tomita M (1992) Upper airway obstruction from a benign goiter. Surg Today 22: 88-90.[Crossref]

9. Agarwal A, Agarwal S, Tewari P, Gupta S, Chand G, etal. (2012) Clinicopathologica profile, airway management, and outcome in huge multinodulargoiters: An institutional experience from and endemic goier region. World Journal of Surgery 36: 755-760.

10. Bartolek D, Frick A (2012) Huge multinodulargoiter with mid trachea obstruction: indication for fiberoptic intubation. Acta Clin Croat 51: 493-498.[Crossref]

Copyright: (C2017 Kusunoki T. This is an open-access article distributed under the terms of the Creative Commons Attribution License, which permits unrestricted use, distribution, and reproduction in any medium, provided the original author and source are credited. 